\title{
ON THE CIRCULAR AREA SIGNATURE FOR GRAPHS
}

\author{
JEFF CALDER ${ }^{\dagger}$ AND SELIM ESEDOGLU ${ }^{\dagger}$
}

\begin{abstract}
The representation of curves by integral invariant signatures is an important step in shape recognition and classification. Integral invariants are preferred over their differential counterparts due to their robustness with respect to noise. However, in contrast to differential invariants of curves, it is currently unknown whether integral signatures offer unique representations of curves. In this article, we prove some results on the uniqueness of the circular area signature. In particular, we study the case for graphs of periodic functions. We show that the circular area signature is unique if taken with respect to parameterization by the $x$-axis. Furthermore, we prove that the true circular area signature (parameterized by arclength) is unique in a neighborhood of constant functions. Finally, we show uniqueness in the special case that the functions of interest agree on an interval of width $2 r$.
\end{abstract}

Key words. Integral invariant signatures, curve descriptors, curves, inverse function theorem, asymptotics

AMS subject classifications. 45Q05, 45M05

1. Introduction. Geometric invariance theory has played an important role in computer vision over the past several decades. The aim of invariance theory in computer vision is to construct functions of an image which are invariant under a group of transformations. In general, the transformations of interest include changes in perspective, lighting and scale. As generic viewpoint invariants do not exist, much attention has been focused on studying invariants to projective transformations in the plane, such as Euclidean or similarity transformations [15]. Such invariants have found applications in shape representation [16, 4], shape matching [3, 13] and object recognition $[19,1]$.

The first invariants used in shape analysis were functions of the curvature of the shape's boundary and are a special case of differential invariants $[6,5]$. Such differential invariants offer simple reconstruction formula and well-known uniqueness results from classical differential geometry [20]. However, as the numerical computations of differential invariants involve computing high order derivatives, they are dominated by the effects of small scale perturbations, such as noise. In an attempt to increase robustness, semi-differential invariants were introduced [17, 21] which involve only first derivatives and a reference point. Although semi-differential invariants are more robust than the curvature-based invariants, they still suffer from susceptibility to noise.

A more principled and robust approach is given by integral invariants which were first introduced by by Manay et al. $[15,14]$ for shape matching and recognition, among other applications in geometry processing (see also $[22,11,8,9,18]$ ). Integral invariant signatures are integral functions of the data instead of differential ones. As such, they retain the Euclidean and similarity invariances of their differential counterparts but are less susceptible to random image fluctuations such as noise. However, the questions of uniqueness of representations and continuity (or even existence) of the reconstruction map are largely unanswered for many integral invariant signatures.

Two particularly interesting integral invariants are the circular and cone area signatures $[9,15]$ (see figure 1). The circular area signature measures the area of the

\footnotetext{
${ }^{\dagger}$ Department of Mathematics, University of Michigan, Ann Arbor, MI 48109. Email: $\{$ jcalder,esedoglu $\} @$ umich.edu
} 


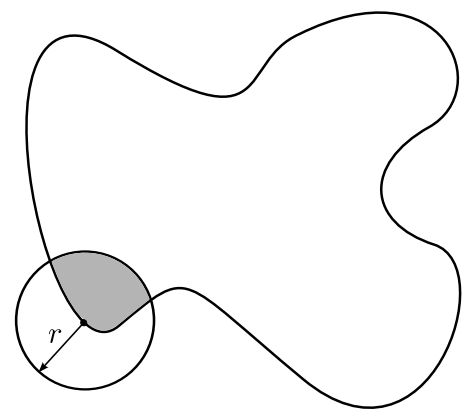

(a) Circular area signature

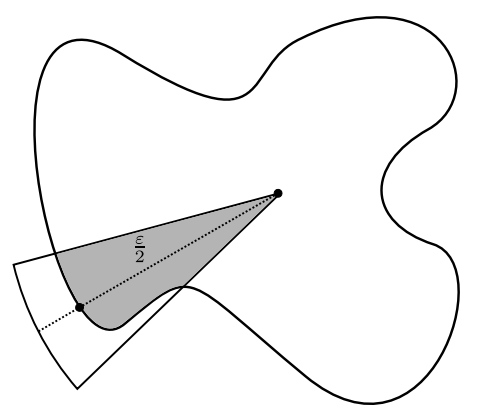

(b) Cone area signature

FIG. 1.1. Depiction of some integral invariant signatures.

intersection of a ball of radius $r>0$ centered on each boundary point with the interior of the object while the cone area signature uses a cone with aperture $\varepsilon>0$ emanating from a common point within the object's interior and centered on each boundary point. The vertex of the cone is commonly chosen to be the centroid of the object. The cone area signature has been thoroughly studied by Fidler et al. [9]. They proved that star-shaped regions are uniquely described by the cone area signature if and only if $\varepsilon / \pi$ is irrational. Furthermore, the inverse map, when it exists, is not continuous.

The circular area signature is perhaps more interesting as it is asymptotically related (as $r \rightarrow 0$ ) to the most popular differential signature, curvature [12]. As such, there is reason to believe that similar uniqueness results to those obtained for curvature may hold for the circular area signature. In fact, recently it has been shown that the circular area signature satisfies a local uniqueness result, weaker than local injectivity, within neighborhoods of circles [2]. However, any kind of global uniqueness result remains elusive. Such a result would be of great interest as it would justify the prominence of the circular area signature in the computer vision literature and advocate its use as a robust invariant signature.

In this work, we study the circular area signature for graphs of periodic functions. Although this is a different problem, it is intimately related to the circular area signature of closed curves. As such, the uniqueness results we prove in this work, aside from being interesting in their own right, indicate that similar results may hold for the case of closed curves.

1.1. Summary of main results. In this work, we study the uniqueness problem for the circular area signature for graphs of periodic functions. To simplify the layout of the paper, we present the main results in this section and postpone the proofs to section 3 after a series of preliminary results.

Let us first fix some notation.

Definition 1.1. For $M=\left(m_{1}, \ldots, m_{4}\right) \in \mathbb{R}_{+}^{4}$ we define

$$
\begin{aligned}
& \Gamma_{M}:=\left\{f \in C^{4}(\mathbb{R}) \mid f(x+2 \pi)\right.=f(x), \forall x \in \mathbb{R}, \\
&\left.\left\|f^{(k)}\right\|_{L^{\infty}(\mathbb{R})} \leq m_{k}, k=1, \ldots, 4, f(0)=0\right\} .
\end{aligned}
$$

We will write $C\left(m_{1}, \ldots, m_{k}\right)$ to denote a positive constant that depends on each of $m_{1}, \ldots, m_{k}$ in a nondecreasing way. Similarly, we will denote by $R\left(m_{1}, \ldots, m_{k}\right)$ a positive constant that depends on each of $m_{1}, \ldots, m_{k}$ in a nonincreasing way. We will often write $C_{k}$ in place of $C\left(m_{1}, \ldots, m_{k}\right)$ and $R_{k}$ in place of $R\left(m_{1}, \ldots, m_{k}\right)$. We will 
write $\|f\|_{\infty}$ in place of $\|f\|_{L^{\infty}(\Omega)}$ when it is clear from the context what the domain of $f$ is. We will use the notation $O_{k}(f)$ to denote a quantity that is bounded by $C_{k}|f|$. We will use the notation $B_{r}(x, y)$ to denote the ball of radius $r$ centered at the point $(x, y)$. We will write $B_{r}$ in place of $B_{r}(0,0)$.

Definition 1.2 (Circular area signature). We define the circular area signature with respect to parametrization via the $x$-axis by

$$
T_{r}(f)(x)=\frac{1}{r^{3}}\left(\int_{B_{r}(x, f(x))} 1_{f}(\mathbf{x}) \mathrm{d} \mathbf{x}-\frac{\pi r^{2}}{2}\right)
$$

where $1_{f}$ denotes the indicator function of the set $\{(x, y) \mid y<f(x)\}$.

With this definition of $T_{r}$, we have that

$$
T_{r}(f)(x)=\frac{1}{3} \kappa_{f}(x)+O\left(r^{2}\right), \text { as } r \rightarrow 0,
$$

where $\kappa_{f}=f^{\prime \prime} /\left(1+f^{\prime 2}\right)^{3 / 2}$ is the curvature of $f$ (see appendix A for proof). Our first result is the following theorem.

TheOrem 1 (Global injectivity). There exists $R=R_{4}$, such that

$$
\left\|f_{1}-f_{2}\right\|_{L^{\infty}(\mathbb{R})} \leq C_{2}\left(1+\frac{1}{r}\left\|f_{1}-f_{2}\right\|_{L^{\infty}(\mathbb{R})}\right)\left\|T_{r}\left(f_{1}\right)-T_{r}\left(f_{2}\right)\right\|_{L^{\infty}(\mathbb{R})},
$$

for all $f_{1}, f_{2} \in \Gamma_{M}, r<R$, and $\left\|T_{r}\left(f_{1}\right)-T_{r}\left(f_{2}\right)\right\|_{L^{\infty}(\mathbb{R})}$ sufficiently small (in terms of $\left.m_{2}\right)$.

This theorem shows that $T_{r}: \Gamma_{M} \rightarrow L^{\infty}(0,2 \pi)$ is injective for $r<R$ and that the inverse satisfies a local stability estimate. We note that the $f(0)=0$ condition in the definition of $\Gamma_{M}$ is merely reflective of the fact that the circular area signature $T_{r}$ is invariant with respect to vertical translation.

We denote by $I_{r}(f)$ the true circular area signature which is parameterized by the arclength parameter of $f$. For $x \in[0,2 \pi]$, we have that $I_{r}(f)(s)=T_{r}(f)(x)$ where

$$
s=\int_{0}^{x} \sqrt{1+f^{\prime}(\xi)^{2}} d \xi .
$$

Before presenting our main results on $I_{r}$, we need the following definitions.

Definition 1.3. For $M=\left(m_{1}, m_{2}, m_{3}, m_{4}\right) \in \mathbb{R}_{+}^{4}, L \in \mathbb{R}_{+}$and $b \in \mathbb{R}$, we define

$$
\Gamma_{M}^{L}:=\left\{f \in \Gamma_{M} \mid \int_{0}^{2 \pi} \sqrt{1+f^{\prime 2}}=L \text { and } f^{\prime}(0)=b\right\} .
$$

Definition 1.4. For $r \in \mathbb{R}^{+}, M=\left(m_{1}, m_{2}\right) \in \mathbb{R}_{+}^{2}, g \in C^{2}(\mathbb{R})$ with $\left\|g^{\prime}\right\|_{L^{\infty}([0,2 r])} \leq$ $m_{1}$ and $\left\|g^{\prime \prime}\right\|_{L^{\infty}([0,2 r])} \leq m_{2}$, we define

$$
\begin{aligned}
\Gamma_{M, r}:=\left\{f \in C^{2}\left(\mathbb{R}_{+}\right) \mid\right. & \left\|f^{\prime}\right\|_{L^{\infty}\left(\mathbb{R}_{+}\right)} \leq m_{1}, \\
& \left.\left\|f^{\prime \prime}\right\|_{L^{\infty}\left(\mathbb{R}_{+}\right)} \leq m_{2}, \quad f=g \text { on }[0,2 r]\right\} .
\end{aligned}
$$

We have the following two theorems regarding $I_{r}$.

Theorem 2 (Local injectivity). There exists $m_{3}>0$ small enough and $R=R_{4}$ such that

$$
\left\|f_{1}-f_{2}\right\|_{L^{\infty}((0,2 \pi))} \leq C_{3}\left\|I_{r}\left(f_{1}\right)-I_{r}\left(f_{2}\right)\right\|_{L^{\infty}(0, L)}
$$


for any $f_{1}, f_{2} \in \Gamma_{M}^{L}, r<R$, and $\left\|I_{r}\left(f_{1}\right)-I_{r}\left(f_{2}\right)\right\|_{\infty}$ sufficiently small.

Theorem 3 (Non-local boundary condition). Let $r<\frac{1}{m_{2}}$. Then $I_{r}: \Gamma_{M, r} \rightarrow$ $L^{\infty}\left(\mathbb{R}_{+}\right)$is injective.

Theorem 2 shows that, provided we remain near constant functions, $I_{r}: \Gamma_{M}^{L} \rightarrow$ $L^{\infty}(0, L)$ is injective for $r<R$ and its inverse satisfies a local stability estimate. The difficulty in proving global injectivity comes from the arclength parametrization which substantially modifies $T_{r}$, making the methods of theorem 1 less effective. In the case of near constant functions, $I_{r}$ can be viewed as a perturbation of $T_{r}$ and the injectivity can be imported from $T_{r}$ yielding theorem 2 . Although we only have a partial result for $I_{r}$, we would argue that parametrization via arclength is somewhat unnatural for the case we are studying as the curves are all graphs of periodic functions. Parametrization via the $x$-axis is much more natural for graphs and so theorem 1 seems to suggest that a global injectivity result for curves may hold, but as we discuss in section 4, the results of this paper cannot be directly applied for arbitrary curves. We should note that there is an additional constraint in $\Gamma_{M}^{L}$ that is not present in the previous theorem, namely $f^{\prime}(0)=b$. This does not have a meaningful interpretation, aside from fixing tangent vectors at the origin, but is necessary due to the fact that we use a continuity result for the second order curvature differential equation and need appropriate initial conditions.

Theorem 3 is somewhat expected. If the functions of interest agree on an interval wider than the ball used for the circular area signature, then we can show injectivity without much of the machinery developed in this paper. This is somewhat less interesting than theorems 1 and 2 as it says little about uniqueness up to geometric transformations (in this case shifts), which is whole purpose of using geometrically invariant signatures.

The proof of theorem 1 relies on linearizing $T_{r}$. We show that the linearization satisfies a maximum principle and use this to bound its inverse. However, since $T_{r}$ is not a $C^{1}$ mapping on any open set in $L^{\infty}$, we cannot directly apply the classical inverse function theorem. Instead, we show that the linearization has quadratic error and use a modified proof of the inverse function theorem to prove local injectivity. Global injectivity follows from the fact that $T_{r}$ is an approximation to curvature, and so by standard ODE theory, if $T_{r}\left(f_{1}\right)=T_{r}\left(f_{2}\right)$ then $f_{1}=f_{2}+O\left(r^{2}\right)$. By choosing $r$ small enough, we can deduce global injectivity from local.

This paper is organized as follows: In section 2 we introduce the linearization of $T_{r}$ and show that the linearization error is quadratic. In section 2.2 we prove the required bounds on the inverse of the linearization. Finally, in section 3 we prove the injectivity results and in section 4 we discuss extensions to the case of closed curves.

2. Linearization of the circular area signature. We now consider the linearization of $T_{r}$. The main result of this section is theorem 2.8 which provides the necessary bound on the inverse of the linearization for the inverse function theorem. We first need some preliminary results on the linearization; in particular, we need to carefully analyze the linearization error, which we do in the next section.

2.1. Linearization error. The main result of this section is theorem 2.3 which shows that the linearization error is quadratic. This is a stronger result than necessary for the classical inverse function theorem. It is necessary here because of the fact that $T_{r}$ does not have a continuous derivative and so the classical inverse function theorem must be subtly modified.

The proposition below is immediate so we omit the proof. 


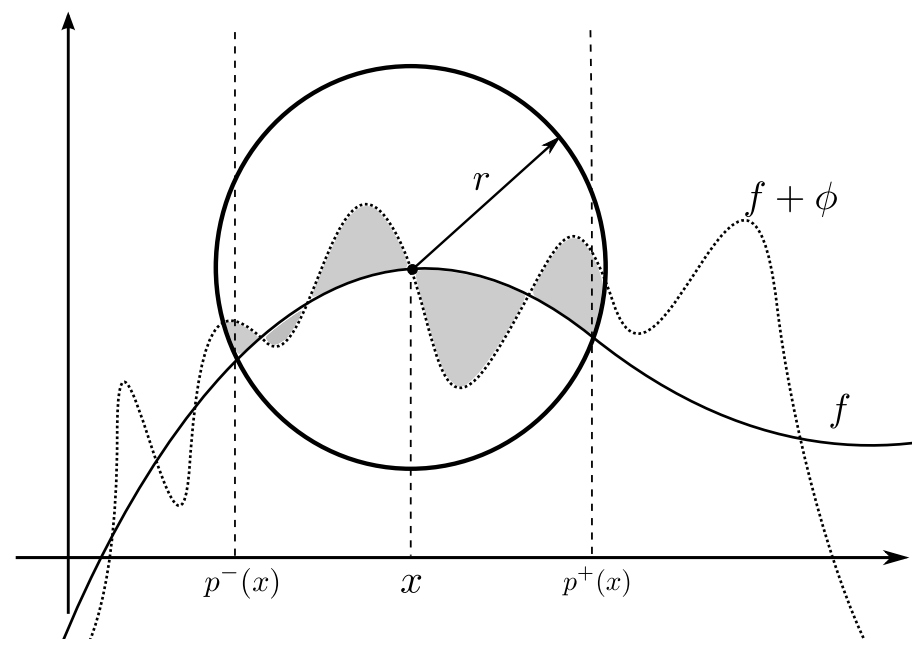

FIG. 2.1. Depiction of some quantities from theorem 2.3 and the definitions of $p^{ \pm}$and $J_{x}$. The shaded area represents $A_{r}(f+\phi)(x)-A_{r}(f)(x)$ and the shaded area to the right of $p^{+}(x)$ and to the left of $p^{-}(x)$ will constitute the linearization error.

Proposition 2.1. Let $f \in C^{2}(\mathbb{R}), r<\left\|f^{\prime \prime}\right\|_{\infty}^{-1}$ and $h \in \mathbb{R}$ with $|h|<r$. Then for each $x \in \mathbb{R}$, the graph of $f+h$ intersects the boundary of $B_{r}(x, f(x))$ in exactly two points.

From here on, we shall always assume that $r<\left\|f^{\prime \prime}\right\|_{\infty}^{-1}$ so that proposition 2.1 always holds. Let us define $p_{f}^{-}(x, h)<p_{f}^{+}(x, h)$ to be the $x$-coordinates of the two points of intersection from proposition 2.1. These are the two distinct solutions, $p$, of

$$
(p-x)^{2}+(f(p)+h-f(x))^{2}=r^{2} .
$$

When $h=0$, we will write $p_{f}^{ \pm}(x)$ in place of $p_{f}^{ \pm}(x, 0)$. When it is clear from the context, we will write $p^{ \pm}$or just $p$ in place of $p_{f}^{ \pm}$. We also set $J_{x}=\left(p_{f}^{-}(x), p_{f}^{+}(x)\right)$ to be the interval from $p_{f}^{-}(x)$ to $p_{f}^{+}(x)$. See figure 2.1 for a depiction of some of these quantities.

For each $f \in C^{2}(\mathbb{R})$ with $\left\|f^{\prime \prime}\right\|_{\infty} \leq 1 / r$, we define the linear map $\mathcal{L}_{f, r}: L^{\infty}(\mathbb{R}) \rightarrow$ $L^{\infty}(\mathbb{R})$ by

$$
\mathcal{L}_{f, r} \phi(x)=\frac{1}{r^{3}}\left(\int_{J_{x}} \phi(y) d y-\left|J_{x}\right| \phi(x)\right) .
$$

As we shall see, $\mathcal{L}_{f, r}$ can be interpreted as the linearization of $T_{r}$ at $f$. We first need an estimate on $p_{f}^{ \pm}(x, h)$.

Lemma 2.2. If $f \in C^{2}(\mathbb{R})$ and $0<\alpha<1$ then

$$
\left|p_{f}^{ \pm}\left(x, h_{1}\right)-p_{f}^{ \pm}\left(x, h_{2}\right)\right| \leq \frac{2}{1-\alpha}\left|h_{1}-h_{2}\right|,
$$

for $\left|h_{1}\right|,\left|h_{2}\right| \leq r(1-\alpha) / 4$ and $r \leq \alpha\left\|f^{\prime \prime}\right\|_{\infty}^{-1}$.

Proof. Fix $x \in \mathbb{R}$. By the definition of $p(h) \equiv p_{f}^{ \pm}(x, h)$ we have

$$
(p(h)-x)^{2}+(f(p(h))+h-f(x))^{2}=r^{2} .
$$


Differentiating in $h$ we have

$$
p^{\prime}(h)=\frac{-(f(p(h))+h-f(x))}{\left.p(h)-x+(f(p(h))+h-f(x)) f^{\prime}(p(h))\right)} .
$$

Translating this problem into the notation of lemma B.1 in the appendix, we have

$$
\hat{\mathbf{n}}(\gamma(\xi))=\frac{1}{r}\langle p(h)-x, f(p(h))+h-f(x)\rangle,
$$

and

$$
\gamma^{\prime}(\xi)=\frac{\left\langle 1, f^{\prime}(p(h))\right\rangle}{\sqrt{1+f^{\prime}(p(h))^{2}}}
$$

where $\gamma$ is the arclength parametrization of $x \mapsto(x, f(x)+h)$ and $\xi$ is such that $\gamma(\xi)$ intersects $B_{r}(x, f(x))$ at $(p(h), f(p(h)+h)$. By lemma B.1 we have that

$$
\left|p^{\prime}(h)\right|=\frac{\mid f(p(h)+h-f(x) \mid}{r \sqrt{1+f^{\prime}(p(h))^{2}}\left|\hat{\mathbf{n}}(\gamma(\xi)) \cdot \gamma^{\prime}(\xi)\right|} \leq \frac{1}{\left|\hat{\mathbf{n}}(\xi) \cdot \gamma^{\prime}(\xi)\right|} \leq \frac{2}{1-\alpha},
$$

for $r \leq \alpha /\left\|f^{\prime \prime}\right\|_{\infty}$ and $|h| \leq r(1-\alpha) / 4$.

TheOREM 2.3. Let $f \in C^{2}(\mathbb{R}), \phi \in C(\mathbb{R})$ and $0<\alpha<1$. Suppose that $r \leq \alpha\left\|f^{\prime \prime}\right\|_{\infty}^{-1}$ and $\|\phi-\phi(x)\|_{L^{\infty}([x-r, x+r])} \leq r(1-\alpha) / 4$. Then

$$
T_{r}(f+\phi)(x)=T_{r}(f)(x)+\mathcal{L}_{f, r} \phi(x)+\frac{1}{r^{3}} \operatorname{err}_{f, r}(\phi)(x),
$$

where

$$
\left|\operatorname{err}_{f, r}(\phi)(x)\right| \leq \frac{C}{1-\alpha}\|\phi-\phi(x)\|_{L^{\infty}([x-r, x+r]}^{2} .
$$

Note that if $\phi \in C^{1}(\mathbb{R})$, then $\left|\operatorname{err}_{f, r}(\phi)(x)\right| \leq \frac{C r^{2}}{1-\alpha}\left\|\phi^{\prime}\right\|_{L^{\infty}([x-r, x+r])}^{2}$.

Proof. Fix an $x \in \mathbb{R}$ and suppose that $\|\phi-\phi(x)\|_{C[x-r, x+r]} \leq r(1-\alpha) / 4$ and $r \leq \alpha\left\|f^{\prime \prime}\right\|_{\infty}^{-1}$. Since $T_{r}$ and $\mathcal{L}_{f, r}$ are invariant under translations, we may assume that $\phi(x)=f(x)=0$. Let $h=\|\phi\|_{L^{\infty}([x-r, x+r])}$ and let $A_{r}(f)(x)$ denote the area inside $B \equiv B_{r}(x, f(x))$ and under $f$. Then we have

$$
T_{r}(f)(x)=\frac{1}{r^{3}}\left(A_{r}(f)(x)-\frac{\pi r^{2}}{2}\right) .
$$

It follows that

$$
\begin{aligned}
\operatorname{err}_{f, r}(\phi)(x) & =A_{r}(f+\phi)(x)-A_{r}(f)(x)-r^{3} \mathcal{L}_{f, r} \phi(x) \\
& =A_{r}(f+\phi)(x)-A_{r}(f)(x)-\int_{J_{x}} \phi(\xi) d \xi
\end{aligned}
$$

Hence, the error consists of the area between $f+\phi$ and $f$ that is either inside $B$ and outside the interval $J_{x}$ or outside $B$ and inside $J_{x}$. See figure 2.1 for a depiction of these regions. Let

$$
\operatorname{err}_{f, r}(\phi)=\operatorname{err}_{f, r}^{+}(\phi)+\operatorname{err}_{f, r}^{-}(\phi)
$$



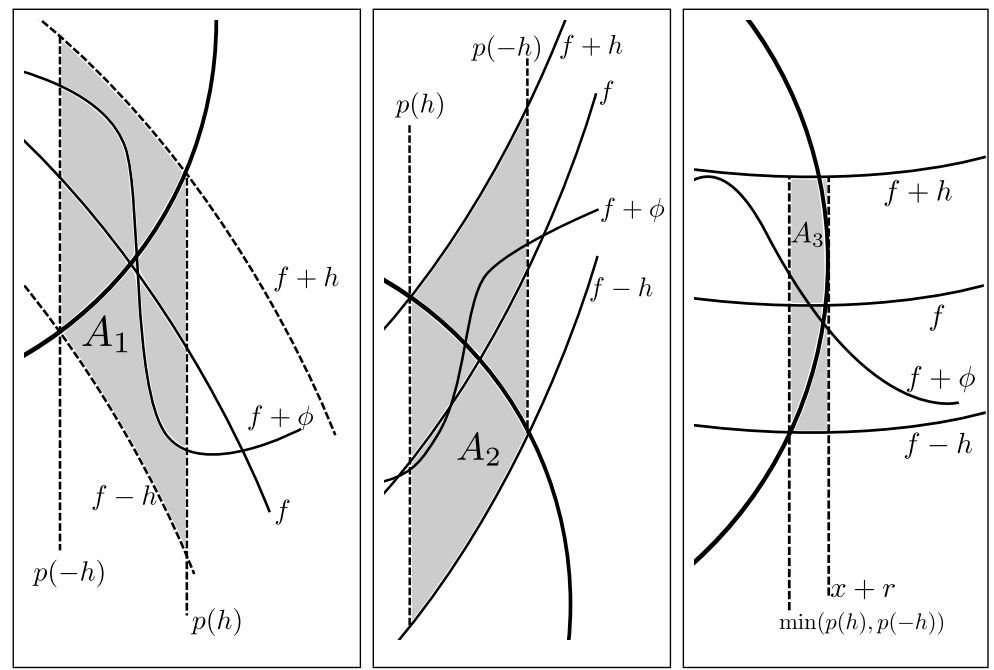

Fig. 2.2. Depiction of the sets $A_{1}, A_{2}$ and $A_{3}$ from theorem 2.3.

where $\operatorname{err}_{f, r}^{+}$and $\operatorname{err}_{f, r}^{-}$are the contributions to the error from the intervals $(x, x+r)$ and $(x-r, x)$ respectively. We first consider $\operatorname{err}_{f, r}^{+}(\phi)$, the error contributed from the right side of $B$, the other case being similar. Fix $x$ and let $p(h) \equiv p_{f}^{+}(x, h)$. There are three qualitatively different ways in which $f-h$ and $f+h$ can intersect the right side of $B$. The first case we will consider is when they both pass through the lower hemisphere, in which case we have $f(p(h)), f(p(-h)) \leq 0$. This implies that $p(-h) \leq p(h)$. Now define

$$
A_{1} \equiv\left\{\left(x_{1}, x_{2}\right)\left|p(-h) \leq x_{1} \leq p(h), \quad\right| f\left(x_{1}\right)-x_{2} \mid \leq h\right\} .
$$

This is a tube above the interval $(p(-h), p(h))$ centered around $f$ and bounded by $f+h$ above and $f-h$ below. The error that contributes to $\operatorname{err}_{f, r}^{+}$is completely contained inside $A_{1}$. See figure 2.2 for a depiction of the region $A_{1}$ as well as $A_{2}$ and $A_{3}$ which are defined below. By lemma 2.2 we have that

$$
\left|\operatorname{err}_{f, r}^{+}(\phi)(x)\right| \leq\left|A_{1}\right| \leq 2 h|p(h)-p(-h)| \leq \frac{C h^{2}}{1-\alpha} .
$$

The second case is when $f+h$ and $f-h$ both pass through the upper hemisphere, in which case $f(p(h)), f(p(-h) \geq 0$ and $p(h) \leq p(-h)$. Then the error is contained in the region

$$
A_{2} \equiv\left\{\left(x_{1}, x_{2}\right)\left|p(h) \leq x_{1} \leq p(-h), \quad\right| f\left(x_{1}\right)-x_{2} \mid \leq h\right\},
$$

and we get an identical conclusion. The final case is when $f+h$ passes through the upper hemisphere and $f-h$ passes through the lower one. Here we have $f(p(h))>$ $0>f(p(-h))$ and we have no knowledge of the ordering of $p(-h)$ and $p(h)$. However, we do know that the error contributing to $\operatorname{err}_{f, r}^{+}$is in this case contained in the region

$$
A_{3} \equiv\left\{\left(x_{1}, x_{2}\right)\left|\min (p(h), p(-h)) \leq x_{1} \leq x+r, \quad\right| f\left(x_{1}\right)-x_{2} \mid \leq h\right\} .
$$

Setting $\tilde{h}=-f(x+r)$, we have $p(\tilde{h})=x+r$. Since $\tilde{h} \in(-h, h)$, we have

$$
|x+r-\min (p(h), p(-h))|=|p(\tilde{h})-\min (p(h), p(-h))| \leq \frac{C h}{1-\alpha}
$$


and hence we have that $\left|\operatorname{err}_{f, r}^{+}(\phi)(x)\right| \leq \frac{C h^{2}}{1-\alpha}$. We can now apply the same reasoning to $\operatorname{err}_{f, r}^{-}$and can conclude that $\left|\operatorname{err}_{f, r}(\phi)(x)\right| \leq \frac{C h^{2}}{1-\alpha}$.

REMARK 1. This theorem can be interpreted as stating that $\mathcal{L}_{f, r}$ is the Fréchet derivative of $T_{r}$ at $f$ provided that $r<1 / m_{2}$. The condition $r<1 / m_{2}$ is a sufficient condition for differentiability of $T_{r}$ but certainly not necessary. One can show that $T_{r}$ is differentiable at $f$ provided each ball $B_{r}(x, f(x))$ intersects $f$ in exactly two points. The condition $r \leq \alpha / m_{2}$ provides us with uniform estimates on the linearization error for all $f \in \Gamma_{M}$ in terms of $\alpha$. If it is not explicitly stated, we will hereafter assume that $r<1 / m_{2}$.

2.2. Estimates for $\mathcal{L}_{f, r}$. The main result of this section is theorem 2.8. This provides the bound on the inverse of $\mathcal{L}_{f, r}$ required to use the inverse function theorem in section 3. The main tool used in the proof is the maximum principle for $\mathcal{L}_{f, r}$ (lemma 2.4). In order to use the maximum principle in the classical way to bound the inverse of an operator, we need to prove the existence of a function $\eta$ with $\mathcal{L}_{f, r} \eta(x) \geq 1$ for all $x$. For this, we require lemma 2.6 establishing the asymptotic behavior of $\mathcal{L}_{f, r}$ as $r \rightarrow 0$. The proof of lemma 2.6 is basic, but tedious, and so it is postponed to appendix B.

We establish first the non-local maximum principle for the operator $\mathcal{L}_{f, r}$.

Lemma 2.4 (Maximum principle). Let $f \in \Gamma_{M}$ and $\phi \in C(\mathbb{R})$. If $\mathcal{L}_{f, r} \phi(x) \geq 0$ for all $x \in[0,2 \pi]$ then

$$
\max _{x \in[0,2 \pi]} \phi(x) \leq \max _{x \in\left[p^{-}(0), 0\right] \cup\left[2 \pi, 2 \pi+p^{+}(0)\right]} \phi(x) .
$$

Proof. Suppose that $\mathcal{L}_{f, r} \phi(x) \geq 0$ for all $x \in[0,2 \pi]$ and let $x^{*} \in\left[p^{-}(0), 2 \pi+p^{+}(0)\right]$ satisfy

$$
\phi\left(x^{*}\right)=\max _{x \in\left[p^{-}(0), 2 \pi+p^{+}(0)\right]} \phi(x) .
$$

Note that $p^{+}(0)=p^{+}(2 \pi)$ as $f$ is $2 \pi$-periodic. Assume that $x^{*} \in(0,2 \pi)$. Since $\mathcal{L}_{f, r} \phi\left(x^{*}\right) \geq 0$, we have

$$
\phi\left(x^{*}\right) \leq \frac{1}{p^{+}\left(x^{*}\right)-p^{-}\left(x^{*}\right)} \int_{p^{-}\left(x^{*}\right)}^{p^{+}\left(x^{*}\right)} \phi(y) d y .
$$

It follows that $\phi(x)=\phi\left(x^{*}\right)$ for all $x \in\left[p^{-}\left(x^{*}\right), p^{+}\left(x^{*}\right)\right]$. By iterating this argument, we conclude that $\phi$ is constant on $[0,2 \pi]$ and the result follows.

It is useful to isolate the follow proposition as it is used in lemma 2.6 and theorem 2.8 .

Proposition 2.5. Let $f \in \Gamma_{M}$. Then for every $x \in \mathbb{R}$, we have

$$
\begin{aligned}
& p_{f}^{+}(x)=x+\frac{r}{\sqrt{1+f^{\prime}(x)^{2}}}-\frac{f^{\prime}(x) f^{\prime \prime}(x)}{2\left(1+f^{\prime}(x)^{2}\right)^{2}} r^{2}+O_{3}\left(r^{3}\right), \\
& p_{f}^{-}(x)=x-\frac{r}{\sqrt{1+f^{\prime}(x)^{2}}}-\frac{f^{\prime}(x) f^{\prime \prime}(x)}{2\left(1+f^{\prime}(x)^{2}\right)^{2}} r^{2}+O_{3}\left(r^{3}\right),
\end{aligned}
$$


Proof. It is easy to see that the osculating circle of $f$ at $x$ intersects the ball $B_{r}(x, f(x))$ at the $x$-coordinates

$$
\begin{aligned}
& x \pm \frac{r \sqrt{1-r^{2} \kappa^{2} / 4}}{\sqrt{1+f^{\prime}(x)^{2}}}-\frac{r^{2} \kappa f^{\prime}(x)}{2 \sqrt{1+f^{\prime}(x)^{2}}} \\
& =x \pm \frac{r}{\sqrt{1+f^{\prime}(x)^{2}}}-\frac{r^{2} f^{\prime}(x) f^{\prime \prime}(x)}{2\left(1+f^{\prime}(x)^{2}\right)^{2}}+O_{2}\left(r^{3}\right) .
\end{aligned}
$$

Noting that the osculating circle approximates $f$ with an error of $O_{3}\left(r^{3}\right)$ completes the proof.

Lemma 2.6 gives an asymptotic representation of $\mathcal{L}_{f, r}$ as $r \rightarrow 0$. The proof is tedious, but comprised of basic calculations, and is postponed to appendix B.

Lemma 2.6. For every $\phi \in C^{3}(\mathbb{R})$, we have

$$
\mathcal{L}_{f, r} \phi(x)=\frac{1}{3\left(1+f^{\prime}(x)^{2}\right)^{3 / 2}} \phi^{\prime \prime}(x)-\frac{f^{\prime}(x) f^{\prime \prime}(x)}{\left(1+f^{\prime}(x)^{2}\right)^{5 / 2}} \phi^{\prime}(x)+g_{\phi}(x) r,
$$

where $\left|g_{\phi}(x)\right| \leq C_{3}\left(\left\|\phi^{\prime}\right\|_{L^{\infty}([x-r, x+r])}+\left\|\phi^{\prime \prime}\right\|_{L^{\infty}([x-r, x+r])}+\left\|\phi^{\prime \prime \prime}\right\|_{L^{\infty}([x-r, x+r]]}\right)$ for all $x \in \mathbb{R}$.

Lemma 2.7. There exists $\eta \in C^{\infty}(\mathbb{R})$ with $\|\eta\|_{C^{3}(\mathbb{R})} \leq C_{2}{ }^{1}$ such that

$$
\mathcal{L}_{f, r} \eta(x) \geq 1,
$$

for all $x \in[0,2 \pi], r<R=R_{3}$ and $f \in \Gamma_{M}$.

Proof. Set $\eta(x)=e^{\beta x}$. By lemma 2.6 and proposition 2.5 we have

$$
\mathcal{L}_{f, r} \eta(x)-r g_{\eta}(x)=\frac{\beta e^{\beta x}}{3\left(1+f^{\prime}(x)^{2}\right)^{3 / 2}}\left(\beta-\frac{3 f^{\prime}(x) f^{\prime \prime}(x)}{1+f^{\prime}(x)^{2}}\right) .
$$

Now choose $\beta>0$ large enough so that

$$
\mathcal{L}_{f, r} \eta(x)-r g_{\eta}(x) \geq 2,
$$

for all $x \in[0,2 \pi]$ and $f \in \Gamma_{M}$. Note that $\beta=\beta\left(m_{1}, m_{2}\right)$ and so $\|\eta\|_{C^{3}(\mathbb{R})} \leq C_{2}$ and $\left\|g_{\eta}\right\|_{\infty} \leq C_{3}$. Now choose $R=R_{3}$ small enough so that for $r<R$, we have $\mathcal{L}_{f, r} \eta(x) \geq 1$ for all $x \in[0,2 \pi]$.

We are now able to prove the main result of this section.

TheOREM 2.8. There exists $R=R_{3}$ such that

$$
\|\phi\|_{\infty} \leq C_{2}\left\|\mathcal{L}_{f, r} \phi\right\|_{\infty}
$$

for all $r<R$, all $2 \pi$-periodic $\phi \in C(\mathbb{R})$ with $\phi(0)=0$, and all $f \in \Gamma_{M}$.

Proof. Let $\bar{\phi} \in L^{\infty}(\mathbb{R})$ be $2 \pi$-periodic with $\bar{\phi}(0)=0$ and let

$$
\phi=\bar{\phi}-\min _{x \in[0,2 \pi]} \bar{\phi}(x) \text {. }
$$

Note that $\|\bar{\phi}\|_{\infty} \leq\|\phi\|_{\infty}$ and $\mathcal{L}_{f, r} \bar{\phi}=\mathcal{L}_{f, r} \phi$. So it is enough to prove the estimate for $\phi$. We may also assume without loss of generality that

$$
\phi(0)=\min _{x \in[0,2 \pi]} \phi(x)=0
$$

\footnotetext{
${ }^{1}$ The $C^{k}$-norm is defined by $\|f\|_{C^{k}(\mathbb{R})}=\sum_{j=0}^{k}\left\|f^{(j)}\right\|_{L^{\infty}(\mathbb{R})}$ for any nonnegative integer $k$.
} 
Let $\psi=\mathcal{L}_{f, r} \phi$ and let $R$ and $\eta$ be as in lemma 2.7. Fix $r<R$ and note that

$$
\mathcal{L}_{f, r}\left(\phi+\|\psi\|_{\infty} \eta\right)(x)=\psi(x)+\|\psi\|_{\infty} \mathcal{L}_{f, r} \eta(x) \geq \psi(x)+\|\psi\|_{\infty} \geq 0
$$

for all $x \in[0,2 \pi]$. By lemma 2.4 we have

$$
\begin{aligned}
\|\phi\|_{\infty}=\max _{x \in[0,2 \pi]} \phi(x) & \leq \max _{x \in\left[p^{-}(0), 0\right] \cup\left[2 \pi, 2 \pi+p^{+}(0)\right]}\left(\phi(x)+\|\psi\|_{\infty} \eta(x)\right) \\
& \leq \max _{x \in\left[p^{-}(0), p^{+}(0)\right]} \phi(x)+C_{2}\|\psi\|_{\infty} \\
& =\|\phi\|_{L^{\infty}\left(J_{0}\right)}+C_{2}\|\psi\|_{\infty} .
\end{aligned}
$$

If we were to have $\|\phi\|_{L^{\infty}\left(J_{0}\right)} \leq \frac{7}{8}\|\phi\|_{\infty}$, then we would be done, so suppose that $\|\phi\|_{L^{\infty}\left(J_{0}\right)}>\frac{7}{8}\|\phi\|_{\infty}$. Hence there exists $\bar{x} \in J_{0}$ such that

$$
\phi(\bar{x})>\frac{7}{8}\|\phi\|_{\infty}
$$

Let

$$
L \phi(x):=\phi(x)-\frac{1}{\left|J_{x}\right|} \int_{J_{x}} \phi(\xi) d \xi .
$$

Then $\mathcal{L}_{f, r} \phi(x)=-\frac{\left|J_{x}\right|}{r^{3}} L \phi(x)$. If $L \phi(\bar{x})>\frac{1}{8}\|\phi\|_{\infty}$, then $\|\phi\|_{\infty} \leq C\|L \phi\|_{\infty} \leq$ $C_{1} r^{2}\left\|\mathcal{L}_{f, r} \phi\right\|_{\infty}$ and we are done, so suppose that

$$
L \phi(\bar{x}) \leq \frac{1}{8}\|\phi\|_{\infty}
$$

Then by (2.2) and (2.3), we have

$$
\frac{7}{8}\|\phi\|_{\infty}-\frac{1}{\left|J_{\bar{x}}\right|} \int_{J_{\bar{x}}} \phi(\xi) d \xi<L \phi(\bar{x}) \leq \frac{1}{8}\|\phi\|_{\infty} .
$$

Hence

$$
\frac{1}{\left|J_{\bar{x}}\right|} \int_{J_{\bar{x}}} \phi(\xi) d \xi \geq \frac{3}{4}\|\phi\|_{\infty}
$$

Without loss of generality, we may assume that $\bar{x}>0$ (note that $\bar{x} \neq 0$ ). Then we have that $\left[p^{-}(\bar{x}), \bar{x}\right] \subset J_{0}$. We have

$$
\begin{aligned}
\frac{1}{\left|J_{0}\right|} \int_{J_{0}} \phi(\xi) d \xi & \geq \frac{\left|J_{\bar{x}}\right|}{\left|J_{0}\right|} \frac{1}{\left|J_{\bar{x}}\right|} \int_{p^{-}(\bar{x})}^{\bar{x}} \phi(\xi) d \xi \\
& =\frac{\left|J_{\bar{x}}\right|}{\left|J_{0}\right|}\left(\frac{1}{\left|J_{\bar{x}}\right|} \int_{J_{\bar{x}}} \phi(\xi) d \xi-\frac{1}{\left|J_{\bar{x}}\right|} \int_{\bar{x}}^{p^{+}(\bar{x})} \phi(\xi) d \xi\right) \\
& \geq \frac{\left|J_{\bar{x}}\right|}{\left|J_{0}\right|}\left(\frac{3}{4}-\frac{p^{+}(\bar{x})-\bar{x}}{\left|J_{\bar{x}}\right|}\right)\|\phi\|_{\infty} \\
& =\frac{1}{\left|J_{0}\right|}\left(\frac{1}{4}\left|J_{\bar{x}}\right|+\bar{x}-\frac{1}{2}\left(p^{+}(\bar{x})+p^{-}(\bar{x})\right)\right)\|\phi\|_{\infty} .
\end{aligned}
$$

By proposition 2.5 we have that

$$
\left|\bar{x}-\frac{1}{2}\left(p^{+}(\bar{x})+p^{-}(\bar{x})\right)\right| \leq C_{2} r^{2} .
$$


Since $\left\|f^{\prime}\right\|_{\infty} \leq m_{1}$, we have that $\left|J_{\bar{x}}\right| \geq r / \sqrt{1+m_{1}^{2}}$. Since $\left|J_{0}\right| \leq 2 r$, we have

$$
\frac{1}{\left|J_{0}\right|} \int_{J_{0}} \phi(\xi) d \xi \geq\left(\frac{1}{8 \sqrt{1+m_{1}^{2}}}-C_{2} r\right)\|\phi\|_{\infty} .
$$

Now choose $r<1 /\left(16 C_{2} \sqrt{1+m_{1}^{2}}\right)$ so that

$$
\|L \phi\|_{\infty} \geq|L \phi(0)|=\frac{1}{\left|J_{0}\right|} \int_{J_{0}} \phi(\xi) d \xi \geq \frac{1}{16 \sqrt{1+m_{1}^{2}}}\|\phi\|_{\infty} .
$$

Hence we have

$$
\|\phi\|_{\infty} \leq C_{1} r^{2}\left\|\mathcal{L}_{f, r} \phi\right\|_{\infty}
$$

3. Injectivity. Before proving our main result, we need a short technical lemma. Lemma 3.1. Let $f_{1}, f_{2} \in \Gamma_{M}$. Then there exists $g_{1}, g_{2} \in \Gamma_{M}$ such that $g_{1}^{\prime}(0)=$ $g_{2}^{\prime}(0)$,

$$
\left\|T_{r}\left(g_{1}\right)-T_{r}\left(g_{2}\right)\right\|_{\infty}=\left\|T_{r}\left(f_{1}\right)-T_{r}\left(f_{2}\right)\right\|_{\infty}
$$

and

$$
\frac{1}{2}\left\|f_{1}-f_{2}\right\|_{\infty} \leq\left\|g_{1}-g_{2}\right\|_{\infty} \leq 2\left\|f_{1}-f_{2}\right\|_{\infty}
$$

Proof. Since $f_{1}-f_{2}$ is $2 \pi$-periodic, it has a maximum and minimum, and hence there exists $y \in[0,2 \pi]$ such that $f_{1}^{\prime}(y)=f_{2}^{\prime}(y)$. Set

$$
g_{1}(x)=f_{1}(x+y)-f_{1}(y), \text { and } g_{2}(x)=f_{2}(x+y)-f_{2}(y) .
$$

Since $T_{r}$ is invariant under vertical shifts, we have

$$
\left\|T_{r}\left(g_{1}\right)-T_{r}\left(g_{2}\right)\right\|_{\infty}=\left\|T_{r}\left(f_{1}\right)-T_{r}\left(f_{2}\right)\right\|_{\infty} .
$$

Now set $\alpha=\left|f_{1}(y)-f_{2}(y)\right| \leq\left\|f_{1}-f_{2}\right\|_{\infty}$. We first have

$$
\left\|g_{1}-g_{2}\right\|_{\infty}=\left\|f_{1}(\cdot+y)-f_{2}(\cdot+y)+f_{2}(y)-f_{1}(y)\right\|_{\infty} \leq 2\left\|f_{1}-f_{2}\right\|_{\infty},
$$

which is one side of the inequality. Now, suppose that $\alpha \leq\left\|f_{1}-f_{2}\right\|_{\infty} / 2$. Then we have

$\left\|f_{1}-f_{2}\right\|_{\infty}=\left\|g_{1}(\cdot-y)-g_{2}(\cdot-y)+f_{1}(y)-f_{2}(y)\right\|_{\infty} \leq\left\|g_{1}-g_{2}\right\|_{\infty}+\frac{1}{2}\left\|f_{1}-f_{2}\right\|_{\infty}$.

Simplifying, we see that $\frac{1}{2}\left\|f_{1}-f_{2}\right\|_{\infty} \leq\left\|g_{1}-g_{2}\right\|_{\infty}$, which is the other side of the inequality in the lemma.

Now suppose that $\alpha \geq\left\|f_{1}-f_{2}\right\|_{\infty} / 2$. Then since $f_{1}(0)=f_{2}(0)=0$, we have $\frac{1}{2}\left\|f_{1}-f_{2}\right\|_{\infty} \leq \alpha=\left|f_{1}(y)-f_{2}(y)\right|=\left|g_{2}(-y)-g_{1}(-y)+f_{1}(0)-f_{2}(0)\right| \leq\left\|g_{1}-g_{2}\right\|_{\infty}$, which again is the other side of the inequality in the lemma.

We now prove our first main result, theorem 1. 
Proof. Let $f_{1}, f_{2} \in \Gamma_{M}$. By lemma 3.1, we may assume that $f_{1}^{\prime}(0)=f_{2}^{\prime}(0)$. If this were not true, then we could use $g_{1}$ and $g_{2}$ from the lemma in place of $f_{1}$ and $f_{2}$. The estimates proved in lemma 3.1 show that the statement of the theorem being true for $f_{1}, f_{2}$ is equivalent to it holding for $g_{1}, g_{2}$.

By the asymptotic expansion of the signature in appendix $\mathrm{A}$, we have that

$$
\frac{f_{i}^{\prime \prime}(x)}{\left(1+f_{i}^{\prime}(x)^{2}\right)^{3 / 2}}=T_{r}\left(f_{i}\right)(x)+O_{4}\left(r^{2}\right), \quad \forall x \in[0,2 \pi], \quad i=1,2 .
$$

By a standard application of Gronwall's inequality (see lemma D.1 in appendix D), we have

$$
\|\phi\|_{\infty},\left\|\phi^{\prime}\right\|_{\infty},\left\|\phi^{\prime \prime}\right\|_{\infty} \leq C_{2}\left\|T_{r}\left(f_{1}\right)-T_{r}\left(f_{2}\right)\right\|_{\infty}+C_{4} r^{2},
$$

where $\phi(x)=f_{1}(x)-f_{2}(x)$. The fact that $\phi(0)=\phi^{\prime}(0)=0$ is used to apply Gronwall's inequality here. By theorem 2.3, we have that $\left\|\operatorname{err}_{f, r}(\phi)\right\|_{\infty} \leq C r^{2}\left\|\phi^{\prime}\right\|_{\infty}^{2}$ for $r \leq$ $1 /\left(2 m_{2}\right)$ and $\left\|\phi^{\prime}\right\|_{\infty} \leq 1 / 4$ by fixing $\alpha=1 / 2$. By (3.1), if we make $\left\|T_{r}\left(f_{1}\right)-T_{r}\left(f_{2}\right)\right\|_{\infty}$ and $R\left(m_{4}\right)$ sufficiently small, then we will have $\left\|\phi^{\prime}\right\|_{\infty} \leq 1 / 4$ for $r<R\left(m_{4}\right)$ and so theorem 2.3 applies. By making $R=R\left(m_{4}\right)$ smaller if necessary, we can use theorem 2.8 , and the interpolation estimate from lemma C.1 to obtain

$$
\begin{aligned}
\|\phi\|_{\infty} & \leq C_{2}\left\|\mathcal{L}_{f_{1}, r} \phi\right\|_{\infty} \\
& =C_{2}\left\|T_{r}\left(f_{2}\right)-T_{r}\left(f_{1}\right)-\frac{1}{r^{3}} \operatorname{err}_{f, r}(\phi)\right\|_{\infty} \\
& \leq C_{2}\left\|T_{r}\left(f_{1}\right)-T_{r}\left(f_{2}\right)\right\|_{\infty}+\frac{C}{r}\left\|\phi^{\prime}\right\|_{\infty}^{2} \\
& \leq C_{2}\left\|T_{r}\left(f_{1}\right)-T_{r}\left(f_{2}\right)\right\|_{\infty}+\frac{C}{r}\left\|\phi^{\prime \prime}\right\|_{\infty}\|\phi\|_{\infty} \\
& \leq C_{2}\left\|T_{r}\left(f_{1}\right)-T_{r}\left(f_{2}\right)\right\|_{\infty}+\frac{C}{r}\left(C_{2}\left\|T_{r}\left(f_{1}\right)-T_{r}\left(f_{2}\right)\right\|_{\infty}+C_{4} r^{2}\right)\|\phi\|_{\infty},
\end{aligned}
$$

for $r<R$ and $\left\|T_{r}\left(f_{1}\right)-T_{r}\left(f_{2}\right)\right\|_{\infty}$ sufficiently small. Hence we have

$$
\|\phi\|_{\infty} \leq C_{2}\left(1+\frac{\|\phi\|_{\infty}}{r}\right)\left\|T_{r}\left(f_{1}\right)-T_{r}\left(f_{2}\right)\right\|_{\infty}+C_{4} r\|\phi\|_{\infty} .
$$

Choosing $R=R\left(m_{4}\right)>0$ smaller, if necessary, completes the proof.

Aside from injectivity, the estimate in theorem 1 gives us a stability result on the reconstruction of $f$ from $T_{r}(f)$. We also note the following corollary.

Corollary 3.2. Assume that the hypotheses of theorem 1 hold and in addition that

$$
\left\|T_{r}\left(f_{1}\right)-T_{r}\left(f_{2}\right)\right\|_{\infty} \leq K r,
$$

for some constant $K>0$. Then we have that

$$
\left\|f_{1}-f_{2}\right\|_{\infty} \leq C_{2}(1+K)\left\|T_{r}\left(f_{1}\right)-T_{r}\left(f_{2}\right)\right\|_{\infty} .
$$

We now aim to prove theorem 2 on the local injectivity of $I_{r}$ by viewing it as a perturbation of $T_{r}$. We will first need some preliminary lemmas. 
Lemma 3.3. We have

$$
\partial_{x} T_{r}(f)(x)=\mathcal{L}_{f, r}\left(f^{\prime}\right)(x)
$$

for $f \in C^{2}(\mathbb{R})$ and $r<\left\|f^{\prime \prime}\right\|_{\infty}^{-1}$.

Proof. For $|h|<1$, let $\phi_{h}(y)=f(y+h)-f(y)$. Note that $\left\|\phi_{h}^{\prime}\right\|_{\infty} \leq m_{1}|h|$ and that $T_{r}(f)(x+h)=T_{r}(f+\phi)(x)$. By theorem 2.3, taking $|h|$ small enough and $r<1 / m_{2}$ we have that

$$
\begin{aligned}
\left|T_{r}(f)(x+h)-T_{r}(f)(x)-\mathcal{L}_{f, r}\left(\phi_{h}\right)(x)\right| & =\left|T_{r}(f+\phi)(x)-T_{r}(f)(x)-\mathcal{L}_{f, r}\left(\phi_{h}\right)(x)\right| \\
& =\frac{1}{r^{3}}\left|\operatorname{err}_{f, r}\left(\phi_{h}\right)(x)\right| \\
& \leq \frac{C h^{2}}{r} .
\end{aligned}
$$

As $\lim _{h \rightarrow 0} \phi_{h} / h=f^{\prime}$, we have that $\partial_{x} T_{r}(f)(x)=\mathcal{L}_{f, r}\left(f^{\prime}\right)(x)$.

COROLlary 3.4. There exists $R=R_{2}$ such that

$$
\left\|\partial_{s} I_{r}(f)\right\|_{\infty} \leq C m_{3}
$$

for $r<R$ and all $f \in \Gamma_{M}$.

Proof. Noting that $\partial_{s} I_{r}(f)=\partial_{x} T_{r}(f) / \sqrt{1+f^{\prime}(x)^{2}}$, we see that

$$
\left\|\partial_{s} I_{r}(f)\right\|_{\infty} \leq\left\|\partial_{x} T_{r}(f)\right\|_{\infty} .
$$

Now, note that

$$
\partial_{x} T_{r}(f)=\mathcal{L}_{f, r}\left(f^{\prime}\right)=\frac{1}{r^{3}}\left(f\left(p^{+}\right)-f\left(p^{-}\right)-\left(p^{+}-p^{-}\right) f^{\prime}(x)\right) .
$$

A Taylor expansion of $f\left(p^{+}\right)$and $f\left(p^{-}\right)$yields

$$
\left|\partial_{x} T_{r}(f)\right| \leq \frac{C m_{2}}{r^{3}}\left|\left(p^{+}-x\right)^{2}-\left(p^{-}-x\right)^{2}\right|+C m_{3} .
$$

By proposition 2.5, we see that $\left(p^{+}-x\right)^{2}-\left(p^{-}-x\right)^{2}=O_{2}\left(r^{3}\right)$, and the corollary follows. $\square$

Note that it follows from the above corollary that $I_{r}(f)$ is a Lipschitz function of the arclength parameter $s$ with a Lipschitz constant that depends on $m_{1}, m_{2}, m_{3}$.

Now, define $S: C^{1}([0,2 \pi]) \rightarrow C^{1}([0,2 \pi])$ by

$$
S(f)=\int_{0}^{x} \sqrt{1+f^{\prime}(\xi)^{2}} d \xi
$$

For any function $f \in \Gamma_{M}^{L}$, we can reparametrize $f$ in terms of its arclength parameter $S(f)$. We will call this reparametrization $\bar{f}$. We can recover the $x$-parameter from $\bar{f}$ as follows. If we let $s(x)=S(f)(x)$, then we have that

$$
(d x)^{2}+\left(\bar{f}^{\prime}(s) d s\right)^{2}=d s^{2} .
$$

Hence we have that $d x=\sqrt{1-\bar{f}^{\prime}(s)^{2}} d s$. This motivates us to define $X: C^{1}([0, L]) \rightarrow$ $C^{1}([0, L])$ by

$$
X(f)=\int_{0}^{s} \sqrt{1-f^{\prime}(\xi)^{2}} d \xi
$$


for any $f \in C^{1}([0, L])$, with $\left\|f^{\prime}\right\|_{\infty}<1$. Then $X(\bar{f})$ is the $x$-parameter of $f$ in terms of the arclength $s$. We have the following identities:

$$
\bar{f}=f \circ X(\bar{f}) \text {, and } f=\bar{f} \circ S(f) .
$$

We now need some estimates on $X$ and $S$.

Lemma 3.5. For $f_{1}, f_{2} \in \Gamma_{M}$, we have

$$
\left\|S\left(f_{1}\right)-S\left(f_{2}\right)\right\|_{\infty} \leq C m_{2}\left\|f_{1}-f_{2}\right\|_{\infty} .
$$

Proof. Set

$$
g(t)=\int_{0}^{x} \sqrt{1+\left(t f_{1}^{\prime}+(1-t) f_{2}^{\prime}\right)^{2}} d \xi .
$$

By considering the Taylor expansion of $g$, we find that

$$
S\left(f_{1}\right)-S\left(f_{2}\right)=g(1)-g(0)=g^{\prime}(0)+O\left(\left\|g^{\prime \prime}\right\|_{\infty}\right) .
$$

Noting that

$$
\begin{aligned}
& g^{\prime}(0)=\int_{0}^{x} \frac{f_{2}^{\prime}\left(f_{1}^{\prime}-f_{2}^{\prime}\right)}{\sqrt{1+f_{2}^{\prime 2}}} d \xi, \text { and } \\
& g^{\prime \prime}(t)=\int_{0}^{x} \frac{\left(f_{1}^{\prime}-f_{2}^{\prime}\right)^{2}}{\sqrt{1+\left(t f_{1}^{\prime}+(1-t) f_{2}^{\prime}\right)^{2}}} d \xi-\int_{0}^{x} \frac{\left(t f_{1}^{\prime}+(1-t) f_{2}^{\prime}\right)^{2}\left(f_{1}^{\prime}-f_{2}^{\prime}\right)^{2}}{\left(1+\left(t f_{1}^{\prime}+(1-t) f_{2}^{\prime}\right)^{2}\right)^{3 / 2}} d \xi
\end{aligned}
$$

we see that $\left\|g^{\prime \prime}\right\|_{\infty} \leq C\left\|f_{1}^{\prime}-f_{2}^{\prime}\right\|_{\infty}^{2}$ and $\left|g^{\prime}(0)\right| \leq C m_{2}\left\|f_{1}-f_{2}\right\|_{\infty}$. Note that the estimate on $g^{\prime}(0)$ involves integrating by parts. By lemma C.1, we have that

$$
\left\|f_{1}^{\prime}-f_{2}^{\prime}\right\|_{\infty}^{2} \leq 2\left\|f_{1}-f_{2}\right\|_{\infty}\left\|f_{1}^{\prime \prime}-f_{2}^{\prime \prime}\right\|_{\infty} \leq C m_{2}\left\|f_{1}-f_{2}\right\|_{\infty}
$$

Hence we have that

$$
\left\|S\left(f_{1}\right)-S\left(f_{2}\right)\right\|_{\infty} \leq C m_{2}\left\|f_{1}-f_{2}\right\|_{\infty} .
$$

Lemma 3.6. Let $f_{1}, f_{2} \in \Gamma_{M}^{L}$. Then we have

$$
\left\|X\left(\bar{f}_{1}\right)-X\left(\bar{f}_{2}\right)\right\|_{\infty} \leq C_{2}\left\|\bar{f}_{1}-\bar{f}_{2}\right\|_{\infty} .
$$

Proof. For any $f \in \Gamma_{M}^{L}$, note that $\left|\bar{f}^{\prime}\right|=\left|f^{\prime} / \sqrt{1+f^{\prime 2}}\right| \leq m_{1} / \sqrt{1+m_{1}^{2}}$. Hence, we have that $\left\|\bar{f}_{i}\right\|_{\infty} \leq 1-\delta$, where

$$
\delta=\frac{\sqrt{1+m_{1}^{2}}-m_{1}}{\sqrt{1+m_{1}^{2}}} .
$$

For the remainder of the proof, we will write $f_{i}$ in place of $\bar{f}_{i}$ to simplify notation. Define

$$
g(t)=\int_{0}^{s} \sqrt{1-\left(t f_{1}^{\prime}+(1-t) f_{2}^{\prime}\right)^{2}} d \xi
$$


Then we have that

$$
X\left(f_{1}\right)-X\left(f_{2}\right)=g(1)-g(0)=g^{\prime}(0)+O\left(\left\|g^{\prime \prime}\right\|_{L^{\infty}(0,1)}\right) .
$$

Noting that

$$
\begin{aligned}
& g^{\prime}(0)=-\int_{0}^{s} \frac{f_{2}^{\prime}\left(f_{1}^{\prime}-f_{2}^{\prime}\right)}{\sqrt{1-f_{2}^{\prime 2}}} d \xi, \text { and } \\
& g^{\prime \prime}(t)=-\int_{0}^{s} \frac{\left(f_{1}^{\prime}-f_{2}^{\prime}\right)^{2}}{\sqrt{1-\left(t f_{1}^{\prime}+(1-t) f_{2}^{\prime}\right)^{2}}} d \xi-\int_{0}^{s} \frac{\left(t f_{1}^{\prime}+(1-t) f_{2}^{\prime}\right)^{2}\left(f_{1}^{\prime}-f_{2}^{\prime}\right)^{2}}{\left(1-\left(t f_{1}^{\prime}+(1-t) f_{2}^{\prime}\right)^{2}\right)^{3 / 2}} d \xi
\end{aligned}
$$

we see that $\left\|g^{\prime \prime}\right\|_{L^{\infty}(0,1)} \leq \frac{C_{1}}{\delta^{3 / 2}}\left\|f_{1}^{\prime}-f_{2}^{\prime}\right\|_{\infty}^{2}$ and $\left|g^{\prime}(0)\right| \leq \frac{C_{2}}{\delta^{1 / 2}}\left\|f_{1}-f_{2}\right\|_{\infty}$. Using the fact that

$$
\left\|f_{1}^{\prime}-f_{2}^{\prime}\right\|_{\infty}^{2} \leq 2\left\|f_{1}-f_{2}\right\|_{\infty}\left\|f_{1}^{\prime \prime}-f_{2}^{\prime \prime}\right\|_{\infty}
$$

we have that

$$
\left\|X\left(f_{1}\right)-X\left(f_{2}\right)\right\|_{\infty} \leq \frac{C_{2}}{\delta^{3 / 2}}\left\|f_{1}-f_{2}\right\|_{\infty} \leq C_{2}\left\|f_{1}-f_{2}\right\|_{\infty}
$$

We can now prove our second main result, theorem 2 .

Proof. Let $f_{1}, f_{2} \in \Gamma_{M}^{L}$, let $c_{1}, c_{2}$ be the curves traced out by the graphs of $f_{1}, f_{2}$, parametrized by arclength and let $\kappa_{1}, \kappa_{2}$ be their curvatures as a function of arclength. Then $c_{1}(0)=c_{2}(0)=(0,0)$ and $c_{1}^{\prime}(0)=c_{2}^{\prime}(0)=(1, b) / \sqrt{1+b^{2}}$. By the asymptotic expansion of $I_{r}$ (appendix A), we have that

$$
\left\|\kappa_{1}-\kappa_{2}\right\|_{L^{\infty}(0, L)} \leq\left\|I_{r}\left(f_{1}\right)-I_{r}\left(f_{2}\right)\right\|_{L^{\infty}(0, L)}+C_{4} r^{2} .
$$

Note that one can obtain the explicit reconstruction formula

$$
\bar{f}_{i}(s)=a+\int_{0}^{s} \sin \left(\arctan (b)+\int_{0}^{\tau} \kappa_{i}(\xi) d \xi\right) d \tau .
$$

It follows that

$$
\left\|\bar{f}_{1}-\bar{f}_{2}\right\|_{L^{\infty}(0, L)} \leq C\left\|\kappa_{1}-\kappa_{2}\right\|_{L^{\infty}(0, L)} \leq C\left\|I_{r}\left(f_{1}\right)-I_{r}\left(f_{2}\right)\right\|_{L^{\infty}(0, L)}+C_{4} r^{2} .
$$

Hence, we have

$$
\begin{aligned}
\left\|f_{1}-f_{2}\right\|_{\infty} & =\left\|f_{1} \circ X\left(\bar{f}_{1}\right)-f_{2} \circ X\left(\bar{f}_{1}\right)\right\|_{L^{\infty}(0, L)} \\
& \leq\left\|f_{1} \circ X\left(\bar{f}_{1}\right)-f_{2} \circ X\left(\bar{f}_{2}\right)\right\|_{L^{\infty}(0, L)}+\left\|f_{2} \circ X\left(\bar{f}_{2}\right)-f_{2} \circ X\left(\bar{f}_{1}\right)\right\|_{L^{\infty}(0, L)} \\
& \leq\left\|\bar{f}_{1}-\bar{f}_{2}\right\|_{\infty}+m_{1}\left\|X\left(\bar{f}_{1}\right)-X\left(\bar{f}_{2}\right)\right\|_{L^{\infty}(0, L)} \\
& \leq C_{2}\left\|I_{r}\left(f_{1}\right)-I_{r}\left(f_{2}\right)\right\|_{L^{\infty}(0, L)}+C_{4} r^{2} .
\end{aligned}
$$

Now note that

$$
\begin{aligned}
\left\|T_{r}\left(f_{1}\right)-T_{r}\left(f_{2}\right)\right\|_{\infty}= & \left\|I_{r}\left(f_{1}\right) \circ S\left(f_{1}\right)-I_{r}\left(f_{2}\right) \circ S\left(f_{2}\right)\right\|_{L^{\infty}(0,2 \pi)} \\
\leq & \left\|I_{r}\left(f_{1}\right) \circ S\left(f_{1}\right)-I_{r}\left(f_{1}\right) \circ S\left(f_{2}\right)\right\|_{L^{\infty}(0,2 \pi)} \\
& +\left\|I_{r}\left(f_{1}\right) \circ S\left(f_{2}\right)-I_{r}\left(f_{2}\right) \circ S\left(f_{2}\right)\right\|_{L^{\infty}(0,2 \pi)} \\
\leq & C m_{3}\left\|S\left(f_{1}\right)-S\left(f_{2}\right)\right\|_{L^{\infty}(0,2 \pi)}+\left\|I_{r}\left(f_{1}\right)-I_{r}\left(f_{2}\right)\right\|_{L^{\infty}(0, L)} \\
\leq & C m_{3} m_{2}\left\|f_{1}-f_{2}\right\|_{L^{\infty}(0, L)}+\left\|I_{r}\left(f_{1}\right)-I_{r}\left(f_{2}\right)\right\|_{L^{\infty}(0, L)} .
\end{aligned}
$$




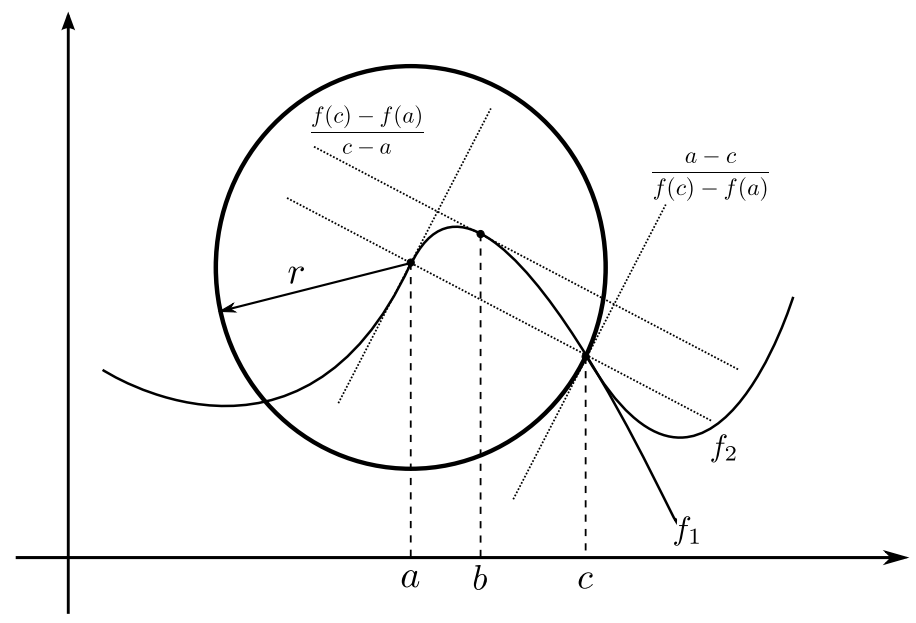

FIG. 3.1. Depiction of some quantities from theorem 3.

Suppose that $\left\|I_{r}\left(f_{1}\right)-I_{r}\left(f_{2}\right)\right\|_{\infty} \leq C r$. Then we have that $\left\|f_{1}-f_{2}\right\|_{\infty} \leq C_{2} r$ for $r<R=R_{4}$ where $R_{4}$ is sufficiently small. It follows that

$$
\left\|T_{r}\left(f_{1}\right)-T_{r}\left(f_{2}\right)\right\|_{L^{\infty}(0,2 \pi)} \leq C_{3} r .
$$

Hence, provided $r \leq R$ for $R=R_{4}$ small enough, we obtain from corollary 3.2 that

$$
\left\|f_{1}-f_{2}\right\|_{\infty} \leq C_{3}\left\|T_{r}\left(f_{1}\right)-T_{r}\left(f_{2}\right)\right\|_{\infty} .
$$

Combining this with (3.6), we have that

$$
\left\|f_{1}-f_{2}\right\|_{\infty} \leq C_{2} m_{2} m_{3}\left\|f_{1}-f_{2}\right\|_{L^{\infty}(0, L)}+C_{3}\left\|I_{r}\left(f_{1}\right)-I_{r}\left(f_{2}\right)\right\|_{L^{\infty}(0, L)} .
$$

By choosing $m_{3}>0$ small enough, we conclude that

$$
\left\|f_{1}-f_{2}\right\|_{\infty} \leq C_{3}\left\|I_{r}\left(f_{1}\right)-I_{r}\left(f_{2}\right)\right\|_{\infty} .
$$

We finally have the proof of theorem 3

Proof. Let $f_{1}, f_{2} \in \Gamma_{M, r}$ such that $I_{r}\left(f_{1}\right) \equiv I_{r}\left(f_{2}\right)$ and take $r<\frac{1}{m_{2}}$. Let

$$
c=\sup \left\{t>0 \quad \mid f_{1}(x)=g_{1}(x) \forall x \in(0, t)\right\} .
$$

and set $J=(0, c)$. For $x \in(0, c)$ we will denote the common value of $f_{1}(x)$ and $f_{2}(x)$ by $f(x)$ Note that we have $2 r \leq c \leq \infty$. Now assume $c<\infty$. Let $a \in[r, c)$ such that $p_{f_{1}}^{+}(a)=p_{f_{2}}^{+}(a)=c$. Since $T_{r}\left(f_{1}\right)(x)=T_{r}\left(f_{2}\right)(x)$ for $x \in(r, c)$ and $r<1 / m_{2}$, we have by lemma 3.3 that $\mathcal{L}_{f_{1}, r}\left(f_{1}^{\prime}\right)(x)=\mathcal{L}_{f_{2}, r}\left(f_{2}^{\prime}\right)(x)$ for $x \in(r, c)$ and hence

$$
\begin{aligned}
& f_{1}\left(p_{f_{1}}^{+}(x)\right)-f_{1}\left(p_{f_{1}}^{-}(x)\right)-\left(p_{f_{1}}^{+}(x)-p_{f_{1}}^{-}(x)\right) f^{\prime}(x) \\
& =f_{2}\left(p_{f_{2}}^{+}(x)\right)-f_{2}\left(p_{f_{2}}^{-}(x)\right)-\left(p_{f_{2}}^{+}(x)-p_{f_{2}}^{-}(x)\right) f^{\prime}(x)
\end{aligned}
$$

for $x \in(r, c)$. Since $f_{1} \equiv f_{2}$ on $(0, c)$, we find that $p_{f_{1}}^{-}(x)=p_{f_{2}}^{-}(x)$ for $x \in(r, c)$. It follows from the above expression that

$$
f^{\prime}(x)\left(p_{f_{1}}^{+}(x)-p_{f_{2}}^{+}(x)\right)=f_{1}\left(p_{f_{1}}^{+}(x)\right)-f_{2}\left(p_{f_{2}}^{+}(x)\right),
$$


for $x \in(r, c)$. Let $I=(a, a+\varepsilon)$ for $\varepsilon>0$. If $p_{f_{1}}^{+}(x)=p_{f_{2}}^{+}(x)$ for all $x \in I$, then from (3.8), we find that $f_{1}\left(p_{f_{1}}^{+}(x)\right)=f_{2}\left(p_{f_{2}}^{+}(x)\right)$ for $x \in I$. This contradicts the definition of $J$ as the largest interval on which $f_{1} \equiv f_{2}$. Hence there exists $x \in I$ such that $p_{f_{1}}^{+}(x) \neq p_{f_{2}}^{+}(x)$. Since this is true for every $\varepsilon>0$, we can find a sequence $x_{1}>x_{2}>x_{3}, \ldots$ such that $x_{n} \rightarrow a, x_{n}>a$, and $p_{f_{1}}^{+}\left(x_{n}\right) \neq p_{f_{2}}^{+}\left(x_{n}\right)$ for all $n$. Note that $p_{f_{1}}^{+}\left(x_{n}\right), p_{f_{2}}^{+}\left(x_{n}\right) \rightarrow c$ as $n \rightarrow \infty$. It follows from (3.8) that

$$
f^{\prime}(a)=\lim _{n \rightarrow \infty} f^{\prime}\left(x_{n}\right)=\lim _{n \rightarrow \infty} \frac{f_{1}\left(p_{f_{1}}^{+}\left(x_{n}\right)\right)-f_{2}\left(p_{f_{2}}^{+}\left(x_{n}\right)\right)}{p_{f_{1}}^{+}\left(x_{n}\right)-p_{f_{2}}^{+}\left(x_{n}\right)} .
$$

Note that the points $\left(p_{f_{1}}^{+}\left(x_{n}\right), f_{1}\left(p_{f_{1}}^{+}\left(x_{n}\right)\right)\right)$ and $\left(p_{f_{2}}^{+}\left(x_{n}\right), f_{2}\left(p_{f_{2}}^{+}\left(x_{n}\right)\right)\right)$ both lie on the boundary of $B_{r}\left(x_{n}, f\left(x_{n}\right)\right)$ by definition. Hence, the points $\left(y_{n}^{1}, y_{n}^{2}\right)$ and $\left(z_{n}^{1}, z_{n}^{2}\right)$, defined by

$$
y_{n}^{1}=p_{f_{1}}^{+}\left(x_{n}\right)+a-x_{n}, \quad \text { and } y_{n}^{2}=f_{1}\left(p_{f_{1}}^{+}\left(x_{n}\right)\right)+f(a)-f\left(x_{n}\right),
$$

and

$$
z_{n}^{1}=p_{f_{2}}^{+}\left(x_{n}\right)+a-x_{n}, \text { and } z_{n}^{2}=f_{2}\left(p_{f_{2}}^{+}\left(x_{n}\right)\right)+f(a)-f\left(x_{n}\right),
$$

lie on the boundary of $B_{r}(a, f(a))$. Furthermore, $\left(y_{n}^{1}, y_{n}^{2}\right),\left(z_{n}^{1}, z_{n}^{2}\right) \rightarrow(c, f(c))$ as $n \rightarrow \infty$. It follows that

$$
f^{\prime}(a)=\lim _{n \rightarrow \infty} \frac{y_{n}^{2}-z_{n}^{2}}{y_{n}^{1}-z_{n}^{1}}=\frac{a-c}{f(c)-f(a)},
$$

the final quantity being precisely the slope of the tangent line to $B_{r}(a, f(a))$ at $(c, f(c))$. See figure 3.1 for a depiction of some of these quantities. Note that since $f^{\prime}$ is continuous and $a \neq c$, we cannot have $f(c)=f(a)$ nor can we have $f^{\prime}(a)=0$. Now, by the mean value theorem, there exists $b \in(a, c)$ such that

$$
f^{\prime}(b)=\frac{f(c)-f(a)}{c-a} .
$$

Noting that

$$
\left|f^{\prime}(b)-f^{\prime}(a)\right|=\frac{r^{2}}{|c-a||f(c)-f(a)|} \geq 1
$$

we have

$$
1 \leq\left|f^{\prime}(b)-f^{\prime}(a)\right| \leq \int_{a}^{b}\left|f^{\prime \prime}(\xi)\right| d \xi \leq r m_{2} .
$$

Hence $r \geq 1 / m_{2}$ which is a contradiction.

4. Discussion and extensions. It is natural to ask if the results in this paper can be extended to closed planar curves. To this end, we consider a simple closed smooth curve $\gamma$ of unit length parametrized by arclength. We define

$$
I_{r}(\gamma)(s)=\operatorname{area}\left(B_{r}(\gamma(s)) \cap \bar{\gamma}\right)
$$


where $\bar{\gamma}$ denotes the interior of $\gamma$. Now, consider the curve $\gamma+\phi \mathbf{n}$, where $\mathbf{n}$ is the unit normal to $\gamma$ and $\phi: \mathbb{S}^{1} \rightarrow \mathbb{R}$ is a smooth normal perturbation. Then we can define

$$
T_{r}(\gamma+\phi \mathbf{n})=\operatorname{area}\left(B_{r}(\gamma(s)+\phi(s) \mathbf{n}) \cap \overline{\gamma+\phi \mathbf{n}}\right) .
$$

Hence $T_{r}$ is the circular area invariant parametrized by the arclength parameter of $\gamma$. One can then show that the linearization of $T_{r}$ is given by

$$
\mathcal{L}_{\gamma, r} \phi(s)=\int_{J_{s}} \phi(\xi) d \xi-g(s) \phi(s),
$$

where $J_{s}=\left(p^{-}(s), p^{+}(s)\right)$ with $p^{ \pm}(s)$ the arclength coordinates of the points of intersection of $B_{r}(\gamma(s))$ and $\gamma$ and $g(s)$ is the length of the projection of $\gamma\left(p^{+}(s)\right)-\gamma\left(p^{-}(s)\right)$ onto the tangent line, i.e.,

$$
g(s)=\left\langle\gamma\left(p^{+}(s)\right)-\gamma\left(p^{-}(s)\right), \gamma^{\prime}(s)\right\rangle
$$

Note that we are assuming that $B_{r}(\gamma(s))$ intersects $\gamma$ in exactly two points for all $s$. Since $\left|J_{s}\right| \geq 2 r \geq g(s)$, and equality cannot hold for all $s$ if $\gamma$ is closed, we see that $\mathcal{L}_{\gamma, r}$ does not satisfy a maximum principle. This is the main difference between the case of closed curves and periodic functions. In the special case that $\gamma$ is a circle, $\mathcal{L}_{\gamma, r}$ is a constant coefficient linear operator and its kernel can be analyzed via Fourier analysis in order to prove a local uniqueness result [2]. In the general case, it is not clear how one would study the kernel of $\mathcal{L}_{\gamma, r}$ and this is perhaps the biggest obstacle in generalizing these results to closed curves.

The other obstacle is the same as the one encountered in this paper, that is, how can we extend injectivity from $T_{r}$ to $I_{r}$. One can easily see that the arclength of $\gamma+\phi \mathbf{n}$, call it $s_{\phi}$, can be written in terms of the arclength parameter of $\gamma$, call it $s$, as follows:

$$
s_{\phi}(s)=\int_{0}^{s} \sqrt{\left(1+\kappa_{\gamma}(s) \phi(s)\right)^{2}+\phi^{\prime}(s)^{2}} d s,
$$

where $\kappa_{\gamma}$ is the curvature of $\gamma$. Then we can write

$$
T_{r}(\gamma+\phi \mathbf{n})(s)=I_{r}(\gamma+\phi \mathbf{n})\left(s_{\phi}(s)\right)
$$

By differentiating with the chain rule, one can see that the linearization of $I_{r}$ is given by

$$
D I_{r} \phi(s)=\mathcal{L}_{\gamma, r} \phi(s)-\left(\partial_{s} I_{r}(\gamma)\right)(s) \int_{0}^{s} \kappa_{\gamma}(\xi) \phi(\xi) d \xi .
$$

Even if the kernel of $\mathcal{L}_{\gamma, r}$ were completely characterized, it is unclear how that knowledge would help one study the kernel of $D I_{r}$, except in the special case where $\partial_{s} I_{r}$ is zero or sufficiently small. The special case of a circle, where $\partial_{s} I_{r} \equiv 0$ has been thoroughly studied [2]. We note that $D I_{r}$ first appeared in [2], but in a different form.

Acknowledgments. We would like to thank the anonymous referees whose detailed comments and suggestions greatly improved the presentation of the paper. 
[1] R. Alferez and Y. F. WAng, Geometric and illumination invariants for object recognition, Pattern Analysis and Machine Intelligence, IEEE Transactions on, 21 (1999), pp. 505-536.

[2] M. Bauer, T. Fidler, And M. Grasmair, Local uniqueness of the circular integral invariant, Arxiv preprint arXiv:1107.4257, (2012).

[3] S. Belongie, J. Malik, and J. Puzicha, Shape matching and object recognition using shape contexts, IEEE Transactions on Pattern Analysis and Machine Intelligence, 24 (2002), pp. 509-522.

[4] A. Bengtsson And J. O. EkLundh, Shape representation by multiscale contour approximation, IEEE Transactions on Pattern Analysis and Machine Intelligence, 13 (1991), pp. 85-93.

[5] Mireille Boutin, Numerically invariant signature curves, Int. J. Comput. Vision, 40 (2000), pp. $235-248$.

[6] E. Calabi, P. J. Olver, C. Shakiban, A. Tannenbaum, and S. Haker, Differential and numerically invariant signature curves applied to object recognition, International Journal of Computer Vision, 26 (1998), pp. 107-135.

[7] L. C. Evans, Partial differential equations, vol. 19 of Graduate Studies in Mathematics, American Mathematical Society, 1998.

[8] T. Fidler, M. Grasmair, H. Pottmann, and O. Scherzer, Inverse problems of integral invariants and shape signatures, FSP 092: Joint Research Program of Industrial Geometry, (2007).

[9] T. Fidler, M. Grasmair, and O. Scherzer, Identifiability and reconstruction of shapes from integral invariants, Inverse Probl. Imaging, 2 (2008), pp. 341-354.

[10] R. S. Hamilton, The inverse function theorem of Nash and Moser, American Mathematical Society, 7 (1982).

[11] Q. X. Huang, S. Flöry, N. Gelfand, M. Hofer, and H. Pottmann, Reassembling fractured objects by geometric matching, ACM Transactions on Graphics (TOG), 25 (2006), pp. 569578.

[12] D. Hulin and M. Troyanov, Mean curvature and asymptotic volume of small balls, American Mathematical Monthly, (2003), pp. 947-950.

[13] S. Z. LI, Shape matching based on invariants, vol. 6 of Progress in Neural Networks, Intellect Books, shape recognition ed., 1999, pp. 203-228. volume 6.

[14] S. Manay, D. Cremers, B. W. Hong, A. J. Yezzi, and S. Soatto, Integral invariants for shape matching, IEEE Transactions on Pattern Analysis and Machine Intelligence, (2006), pp. $1602-1618$.

[15] S. Manay, B. W. Hong, A. Yezzi, And S. Soatto, Integral invariant signatures, Computer Vision-ECCV 2004, (2004), pp. 87-99.

[16] D. Mumford, A. Latto, and J. Shah, The represenatation of shape, in IEEE Workshop on Computer Vision, 1984, pp. 183-191.

[17] T. Pajdla AND L. VAN GoOL, Matching of 3-d curves using semi-differential invariants, in Computer Vision, 1995. Proceedings., Fifth International Conference on, jun 1995, pp. 390395.

[18] H. Pottmann, J. Wallner, Q. X. Huang, and Y. L. Yang, Integral invariants for robust geometry processing, Computer Aided Geometric Design, 26 (2009), pp. 37-60.

[19] T. H. REISs, Recognizing planar objects using invariant image features, Lecture notes in computer science, Springer-Verlag, 1993.

[20] D. J. Struik, Lectures on classical differential geometry, Dover Publications, 1988.

[21] L. Van Gool, T. Moons, E. Pauwels, and A. Oosterlinck, Semi-differential invariants, Geometric Invariance in Computer Vision, MIT, Cambridge, 1992, pp. 193-214.

[22] Y. L. Yang, Y. K. LaI, S. M. HU, And H. Pottmann, Robust principal curvatures on multiple scales, in Proceedings of the fourth Eurographics symposium on Geometry processing, 2006, pp. 223-226.

Appendix A. Asymptotic expansion of signature. We now compute the asymptotic expansion of the area invariant $A_{r}$ for a $C^{4}$ curve $c$. We may assume that the curve $c$ is the graph of a $C^{4}$ function $f$ with $f^{\prime}(0)=f(0)=0$. Let $m_{k}=$ $\left\|f^{(k)}\right\|_{L^{\infty}(-r, r)}$ for $k=1,2,3,4$ and note that $\kappa=f^{\prime \prime}(0)$. Let $p^{-}(r)<p^{+}(r)$ be the $x$-coordinates of the points of intersection of $B_{r}$ with $f$. We will assume that $r<1 / m_{2} \leq 1 / \kappa_{\max }$.

Theorem A.1. $A_{r}=\frac{\pi r^{2}}{2}+\frac{1}{3} \kappa r^{3}+O_{4}\left(r^{5}\right)$. 
Proof. For simplicity, we assume that $f\left(p^{ \pm}\right)<0$. The proofs for the other cases proceed identically. Then we have that

$$
A_{r}=\int_{p^{-}}^{p^{+}} f(x)+\sqrt{r^{2}-x^{2}} d x
$$

By proposition 2.5 and the fact that $f^{\prime}(0)=0$ we have

$$
p^{ \pm}= \pm r+O_{3}\left(r^{3}\right)
$$

It follows that

$$
\begin{aligned}
\int_{p^{-}}^{p^{+}} f(x) d x & =\int_{p^{-}}^{p^{+}} \frac{1}{2} \kappa x^{2}+\frac{1}{6} f^{\prime \prime \prime}(0) x^{3} d x+O_{4}\left(r^{5}\right) \\
& =\frac{1}{6} \kappa\left(p^{+^{3}}-p^{-3}\right)+O_{4}\left(r^{5}\right) \\
& =\frac{1}{3} \kappa r^{3}+O_{4}\left(r^{5}\right) .
\end{aligned}
$$

Also, we have

$$
\int_{p^{-}}^{p^{+}} \sqrt{r^{2}-x^{2}} d x=\int_{-r}^{r} \sqrt{r^{2}-x^{2}} d x+\int_{p^{-}}^{-r} \sqrt{r^{2}-x^{2}} d x+\int_{r}^{p^{+}} \sqrt{r^{2}-x^{2}} d x
$$

Hence

$$
\left|\int_{p^{-}}^{p^{+}} \sqrt{r^{2}-x^{2}} d x-\frac{\pi r^{2}}{2}\right| \leq\left|p^{-}+r\right|\left(\sup _{-r \leq x \leq p^{-}} \sqrt{r^{2}-x^{2}}\right)+\left|r-p^{+}\right|\left(\sup _{p^{+} \leq x \leq r} \sqrt{r^{2}-x^{2}}\right) .
$$

Note that the supremum in each case above occurs at $x=p^{ \pm}$so we have

$$
\sqrt{r^{2}-p^{ \pm 2}}=\sqrt{r^{2}-\left(r+O_{3}\left(r^{3}\right)\right)^{2}}=O_{3}\left(r^{2}\right)
$$

Since $\left|r-p^{+}\right|=O_{3}\left(r^{3}\right)$ and $\left|p^{-}+r\right|=O_{3}\left(r^{3}\right)$, we have that

$$
\int_{p^{-}}^{p^{+}} \sqrt{r^{2}-x^{2}} d x=\frac{\pi r^{2}}{2}+O_{3}\left(r^{5}\right)
$$

It follows that

$$
A_{r}=\frac{\pi r^{2}}{2}+\frac{1}{3} \kappa r^{3}+O_{4}\left(r^{5}\right) .
$$

$\square$ We note that a similar result (with $O\left(r^{4}\right)$ error) has already appeared in the literature [12]. The fact that one can improve the error (to $\left.O\left(r^{5}\right)\right)$ by bounding the curve in $C^{4}$ is of critical importance to our main result.

Appendix B. Technical lemmas and proofs. Below is a useful, but standard, lemma on the geometry of curves.

Lemma B.1. Let $\gamma=\left(x_{1}, x_{2}\right)$ be a $C^{2}$ curve in $\mathbb{R}^{2}$ parameterized by arclength with maximum absolute value of curvature $\kappa_{\max }$. For $0<\alpha<1$, let $r \leq \alpha / \kappa_{\max }$ and suppose that $\gamma(0)=(0, h)$ where $|h| \leq r(1-\alpha) / 2$. Let $\xi>0$ be the smallest positive 
number for which $\gamma(\xi)$ intersects the boundary of $B_{r}$. Let $\hat{\mathbf{n}}(x, y)$ be the outward unit normal to $B_{r}$. Then we have

$$
\left|\hat{\mathbf{n}}\left(x_{1}(\xi), x_{2}(\xi)\right) \cdot \gamma^{\prime}(\xi)\right| \geq \frac{1}{2}(1-\alpha) .
$$

Proof. Let $a(s)=x_{1}(s) x_{1}^{\prime}(s)+x_{2}(s) x_{2}^{\prime}(s)$ and suppose $0<s \leq \xi$. Suppose that $r \leq \alpha / \kappa_{\max }$ where $0<\alpha<1$. Then we have

$$
a^{\prime}(s)=1+x_{1}(s) x_{1}^{\prime \prime}(s)+x_{2}(s) x_{2}^{\prime \prime}(s) .
$$

By the Cauchy-Schwarz inequality ${ }^{2}$ we have

$\left|a^{\prime}(s)-1\right|=\left|x_{1}(s) x_{1}^{\prime \prime}(s)+x_{2}(s) x_{2}^{\prime \prime}(s)\right| \leq \sqrt{x_{1}(s)^{2}+x_{2}(s)^{2}} \sqrt{x_{1}^{\prime \prime}(s)^{2}+x_{2}^{\prime \prime}(s)^{2}} \leq r|\kappa(s)|$.

Since $\left|x_{2}^{\prime}(0)\right| \leq 1$, the above bound yields

$$
s-|h|-|a(s)| \leq\left|a(s)-s-h x_{2}^{\prime}(0)\right| \leq \int_{0}^{s}\left|a^{\prime}(\tau)-1\right| d \tau \leq \kappa_{\max } r s \leq \alpha s .
$$

Hence $|a(s)| \geq s(1-\alpha)-|h|$. Now suppose that $|h| \leq \beta r$, with $0<\beta<1$. Noting that $\xi \geq r-|h|$,

$$
\left|\hat{\mathbf{n}}\left(x_{1}(\xi), x_{2}(\xi)\right) \cdot \gamma^{\prime}(\xi)\right|=\frac{1}{r}|a(\xi)| \geq \frac{1}{r}(\xi(1-\alpha)-|h|) \geq 1-\alpha-2 \beta .
$$

Setting $\beta=(1-\alpha) / 4$ completes the proof.

The proof of lemma 2.6 is below.

Proof. Let $a(x)=r\left(1+f^{\prime}(x)^{2}\right)^{-1 / 2}$ and note that we can write

$$
\begin{aligned}
\mathcal{L}_{f, r} \phi(x)= & \frac{1}{r^{3}}\left(\int_{p^{-}(x)}^{p^{+}(x)} \phi(y) d y-\left|J_{x}\right| \phi(x)\right) \\
= & \frac{1}{r^{3}}\left(\int_{x-a(x)}^{x+a(x)} \phi(y) d y-2 a(x) \phi(x)\right) \\
& +\frac{1}{r^{3}}\left(\int_{p^{-}(x)}^{x-a(x)} \phi(y) d y+\int_{x+a(x)}^{p^{+}(x)} \phi(y) d y+\left(2 a(x)-\left|J_{x}\right|\right) \phi(x)\right) \\
= & : A+B .
\end{aligned}
$$

Since $\phi \in C^{3}(\mathbb{R})$, we can expand $\phi$ via a Taylor series

$$
\phi(y)=\phi(x)+(y-x) \phi^{\prime}(x)+\frac{1}{2}(y-x)^{2} \phi^{\prime \prime}(x)+\frac{1}{6} g_{1}(y)(y-x)^{3},
$$

where $\left\|g_{1}\right\|_{L^{\infty}([x-r, x+r])} \leq\left\|\phi^{\prime \prime \prime}\right\|_{L^{\infty}([x-r, x+r])}$. Hence, for $A$ we have

$$
\begin{aligned}
A= & \frac{1}{r^{3}}\left(\int_{x-a(x)}^{x+a(x)} \phi(x)+(y-x) \phi^{\prime}(x)+\frac{1}{2}(y-x)^{2} \phi^{\prime \prime}(x)\right. \\
& \left.+\frac{1}{6} g_{1}(y)(y-x)^{3} d y-2 a(x) \phi(x)\right) \\
= & \frac{1}{3}\left(1+f^{\prime}(x)^{2}\right)^{-3 / 2} \phi^{\prime \prime}(x)+\frac{1}{6 r^{3}} \int_{x-a(x)}^{x+a(x)} g_{1}(y)(y-x)^{3} d y .
\end{aligned}
$$

\footnotetext{
${ }^{2}$ the Cauchy-Schwarz inequality used here is $\left|a_{1} b_{1}+a_{2} b_{2}\right| \leq \sqrt{a_{1}^{2}+a_{2}^{2}} \sqrt{b_{1}^{2}+b_{2}^{2}}$.
} 
For $B$, we expand $\phi$ via a first order Taylor series as

$$
\phi(y)=\phi(x)+(y-x) \phi^{\prime}(x)+\frac{1}{2} g_{2}(y)(y-x)^{2},
$$

where $\left\|g_{2}\right\|_{L^{\infty}([x-r, x+r])} \leq\left\|\phi^{\prime \prime}\right\|_{L^{\infty}([x-r, x+r])}$. Then we have

$$
\begin{aligned}
& \int_{p^{-}(x)}^{x-a(x)} \phi(y) d y+\int_{x+a(x)}^{p^{+}(x)} \phi(y) d y \\
= & \int_{p^{-}(x)}^{x-a(x)} \phi(x)+(y-x) \phi^{\prime}(x)+\frac{1}{2} g_{2}(y)(y-x)^{2} d y \\
& +\int_{x+a(x)}^{p^{+}(x)} \phi(x)+(y-x) \phi^{\prime}(x)+\frac{1}{2} g_{2}(y)(y-x)^{2} d y \\
= & \phi(x)\left(\left|J_{x}\right|-2 a(x)\right)+\frac{1}{2} \phi^{\prime}(x)\left(\left(p^{+}(x)-x\right)^{2}-\left(p^{-}(x)-x\right)^{2}\right) \\
& +\frac{1}{2} \int_{p^{-}(x)}^{x-a(x)} g_{2}(y)(y-x)^{2} d y+\frac{1}{2} \int_{x+a(x)}^{p^{+}(x)} g_{2}(y)(y-x)^{2} d y
\end{aligned}
$$

Hence we have

$$
\begin{aligned}
B= & \frac{1}{r^{3}}\left(\frac{1}{2} \phi^{\prime}(x)\left(\left(p^{+}(x)-x\right)^{2}-\left(p^{-}(x)-x\right)^{2}\right)\right. \\
& \left.+\frac{1}{2} \int_{p^{-}(x)}^{x-a(x)} g_{2}(y)(y-x)^{2} d y+\frac{1}{2} \int_{x+a(x)}^{p^{+}(x)} g_{2}(y)(y-x)^{2} d y\right) .
\end{aligned}
$$

Also, note that by proposition 2.5 we have

$$
\left(p^{+}(x)-x\right)^{2}-\left(p^{-}(x)-x\right)^{2}=-\frac{2 f^{\prime}(x) f^{\prime \prime}(x)}{\left(1+f^{\prime}(x)^{2}\right)^{5 / 2}} r^{3}+O_{3}\left(r^{4}\right) .
$$

So we have

$$
\mathcal{L}_{f, r} \phi=\frac{1}{3}\left(1+f^{\prime}(x)^{2}\right)^{-3 / 2} \phi^{\prime \prime}(x)-\frac{f^{\prime}(x) f^{\prime \prime}(x)}{\left(1+f^{\prime}(x)^{2}\right)^{5 / 2}} \phi^{\prime}(x)+r g_{\phi}(x),
$$

where

$$
\begin{gathered}
g_{\phi}(x)=\frac{1}{6 r^{4}} \int_{x-a(x)}^{x+a(x)} g_{1}(y)(y-x)^{3} d y+\frac{1}{2 r^{4}}\left(\int_{p^{-}(x)}^{x-a(x)} g_{2}(y)(y-x)^{2} d y\right. \\
\left.+\int_{x+a(x)}^{p^{+}(x)} g_{2}(y)(y-x)^{2} d y\right)+C_{3} \phi^{\prime}(x) .
\end{gathered}
$$

An elementary calculation (using proposition 2.5) shows that

$$
\left|g_{\phi}(x)\right| \leq C_{3}\left(\left\|\phi^{\prime}\right\|_{L^{\infty}([x-r, x+r])}+\left\|\phi^{\prime \prime}\right\|_{L^{\infty}([x-r, x+r])}+\left\|\phi^{\prime \prime \prime}\right\|_{L^{\infty}([x-r, x+r])}\right) .
$$

Appendix C. Interpolation. The following interpolation lemma can be found in [10]. We include it here for completeness. 
Lemma C.1 (Interpolation). For any $2 \pi$-periodic $f \in C^{2}(\mathbb{R})$ with $f(x)=0$ for some $x \in[0,2 \pi)$, we have

$$
\left\|f^{\prime}\right\|_{\infty}^{2} \leq 2\|f\|_{\infty}\left\|f^{\prime \prime}\right\|_{\infty}
$$

Proof. Note that if $\left\|f^{\prime \prime}\right\|_{\infty}=0$ or $\|f\|_{\infty}=0$, then $\left\|f^{\prime}\right\|_{\infty}=0$ and so (C.1) is satisfied. So we may assume that $\left\|f^{\prime \prime}\right\|_{\infty}$ and $\|f\|_{\infty}$ are nonzero. For $a, b>0$, define $\bar{f}(x)=a f(b x)$. Then we have that

$$
\left\|\bar{f}^{\prime}\right\|_{\infty}^{2}=a^{2} b^{2}\left\|f^{\prime}\right\|_{\infty}^{2}, \text { and }\|\bar{f}\|_{\infty}\left\|\bar{f}^{\prime \prime}\right\|_{\infty}=a^{2} b^{2}\|f\|_{\infty}\left\|f^{\prime \prime}\right\|_{\infty} .
$$

Hence, $f$ satisfies (C.1) if and only if $\bar{f}$ satisfies (C.1) for any $a, b>0$. Since we can choose $a, b>0$ so that $\left\|\bar{f}^{\prime}\right\|_{\infty}=\left\|\bar{f}^{\prime \prime}\right\|_{\infty}=1$, we may assume that $\left\|f^{\prime}\right\|_{\infty}=\left\|f^{\prime \prime}\right\|_{\infty}=1$ and must show that $\|f\|_{\infty} \geq 1 / 2$. By translating $f$ we may assume that $\sup \left|f^{\prime}\right|$ occurs at the origin. By reflecting $f$ about either axis, we may assume that $f(0) \geq 0$ and $f^{\prime}(0)=\sup \left|f^{\prime}\right|=1$. By the mean value theorem, we have that

$$
f(1)=f(0)+f^{\prime}(0)+\frac{1}{2} f^{\prime \prime}(\xi)
$$

for some $\xi \in[0,1]$. Hence we have

$$
\|f\|_{\infty} \geq f(1) \geq 1-\frac{1}{2}=\frac{1}{2} .
$$

Appendix D. Other estimates.

Lemma D.1. Suppose that $f_{1}, f_{2} \in \Gamma_{M}$ and let

$$
g_{i}(x)=\frac{f^{\prime \prime}(x)}{\left(1+f_{i}^{\prime}(x)^{2}\right)^{3 / 2}}, \quad i=1,2
$$

and $\phi=f_{1}-f_{2}$. Then we have that

$$
\|\phi\|_{\infty},\left\|\phi^{\prime}\right\|_{\infty},\left\|\phi^{\prime \prime}\right\|_{\infty} \leq C\left(m_{2}\right)\left\|g_{1}-g_{2}\right\|_{\infty} .
$$

Proof. Let $a_{i}(x)=\left(1+f_{i}^{\prime}(x)^{2}\right)^{3 / 2}$. Then we have that

$$
\begin{aligned}
f_{1}^{\prime}(x)-f_{2}^{\prime}(x) & =\int_{0}^{x} f_{1}^{\prime \prime}(\xi)-f_{2}^{\prime \prime}(\xi), d \xi \\
& =\int_{0}^{x} a_{1}(\xi)\left(g_{1}(\xi)-g_{2}(\xi)\right)+\left(a_{1}(\xi)-a_{2}(\xi)\right) g_{2}(\xi) d \xi .
\end{aligned}
$$

Hence

$$
\left|\phi^{\prime}(x)\right| \leq C_{1}\left\|g_{1}-g_{2}\right\|_{\infty}+C_{2} \int_{0}^{x}\left|\phi^{\prime}(x)\right| d \xi .
$$

By applying Gronwall's inequality (see [7]), we have

$$
\left|\phi^{\prime}(x)\right| \leq C_{2}\left\|g_{1}-g_{2}\right\|_{\infty}
$$

The other estimates follow immediately. $\square$ 